\title{
Recurrent Encephalopathy with Transverse Myelitis: An Uncommon Presentation of Neurobrucellosis
}

\author{
Ravi K. Anadure ${ }^{1} \quad$ jitesh Goel ${ }^{1} \quad$ S. Sahu ${ }^{2} \quad$ Tushar Vidhale $^{3}$ \\ ${ }^{1}$ Department of Neurology, Command Hospital Air Force (CHAF), \\ Bengaluru, Karnataka, India \\ 2Department of Radiology, Command Hospital Air Force (CHAF), \\ Bengaluru, Karnataka, India \\ ${ }^{3}$ Department of Medicine, Command Hospital Air Force (CHAF), \\ Bengaluru, Karnataka, India
}

J Neurosci Rural Pract 2019;10:703-706

\begin{abstract}
Address for correspondence Ravi K. Anadure, VSM, Department of Medicine and Neurology, Agram Post, Command Hospital Air Force (CHAF), Bengaluru 560007, India (e-mail: ravi.anadure@gmail.com).
\end{abstract}

\begin{abstract}
Keywords

- neurobrucellosis

- myeloradiculopathy

- malta fever

Brucellosis (malta fever) is a zoonotic infection caused by a gram-negative coccobacillus which is a facultative intracellular pathogen. It causes a chronic granulomatous infection, similar in histology to tuberculosis. Brucellosis remains a diagnostic dilemma due to misleading, nonspecific manifestations and increasing trend of unusual presentations. In brucellosis, the nervous system involvement occurs in only 5 to $7 \%$ of untreated patients, and it may manifest with encephalitis, meningoencephalitis, radiculitis, and peripheral and cranial neuropathies. Transverse myelitis is an unusual occurrence. Here, the authors are reporting an unusual case of neurobrucellosis in an elderly male patient who visited multiple hospitals with recurring febrile encephalopathy and paraparesis. The diagnosis was suspected by his occupational history of working as an abattoir worker and was confirmed by the presence of high titers of Brucella immunoglobulin ( $\mathrm{g}$ ) M and $\mathrm{IgG}$ antibodies in the serum. The patient was managed with injection gentamicin for 2 weeks along with oral course of doxycycline and rifampicin for 6 weeks. He made a good clinical recovery and went back to work with mild residual deficits.
\end{abstract}

\section{Introduction}

Brucellosis is an important zoonosis worldwide which is showing reemergence in the past few decades, because of multiple sanitary and socioeconomic reasons. ${ }^{1,2}$ Various case reports suggest that brucellosis is endemic in India but authentic data on its true human prevalence is lacking. ${ }^{3}$ The primary host for brucella are domestic animals like sheep, goat, camel, and the secondary host are humans. ${ }^{4}$

Brucella melitensi is one of the commonest species to cause symptomatic disease in humans. Uncooked meat and unpasteurized dairy products are the usual modes of transmission. ${ }^{3}$ Occupational history is of paramount importance in brucellosis. In endemic areas, farmers, shepherds, veterinarians, and employees in slaughterhouses are commonly affected by brucellosis. ${ }^{4}$ The clinical manifestations of brucellosis may appear in acute, subacute or chronic, and relapsing forms. ${ }^{5,6}$

\section{Case History}

A 62-year-old man, resident of Dharampuri (Tamil Nadu), an old case of diabetes mellitus on irregular treatment, presented with history of febrile delirium and bilateral lower limb weakness of 6-month duration. He had recurrent episodes of high-grade fever with delirium, requiring multiple hospital admissions at nearby private hospitals. Each time he was managed with short courses of intravenous (IV) antibiotics for 5 to 7 days. Along with fever, he also noticed subacute onset of symmetrical weakness of both lower limbs with sensation of tightness in both lower limb and band like sensation at lower border of chest. On admission at our hospital, patient was unable to walk and was wheel-chair bound. He had difficulty in passing urine after first episode of lower limb weakness for which he was catheterized elsewhere (since then catheter was in situ and changed every 3-4 weeks). He gave 
history of constitutional symptoms like malaise, body aches, and arthralgia along with unquantified weight loss in the last 6 months.

On clinical examination, his MMSE (Mini Mental State Examination) was $30 / 30$, speech and fundus examination was normal. He had a Gibbus at T12/L1 spinal level. On motor examination, the tone was increased with grade- 2 spasticity in both lower limbs. Upper limb power was normal, but in the lower limbs, antigravity muscle weakness was present (grade 4/5). All deep tendon reflexes were brisk, and his plantar reflexes were extensors bilaterally. There was a sharp sensory level (75\%) at T7 dermatome for all modalities. There were no cerebellar or extrapyramidal signs. A clinical diagnosis of chronic encephalomyelitis was made and a broad differential was considered, which included tubercular (TB) meningitis with arachnoiditis, multiple myeloma, lymphoma with the central nervous system (CNS) involvement and occult malignancy with CNS metastasis.

His investigation revealed mild anemia $(\mathrm{Hb}=10 \mathrm{~g} \%$ with normocytic normochromic blood picture) and a marginally raised erythrocyte sedimentation rate $(22 \mathrm{~mm}$ fall in first hour). His Mantoux's test was nonreactive. His cerebrospinal fluid (CSF) biochemistry and cytology were normal. There were no malignant cells on CSF cytospin. His routine investigation which included liver and renal function test, blood coagulation studies, electrolytes, and urine examination were all within normal limits. No growth in blood and urine cultures was noted after 72 hours incubation. Serologic tests for hepatitis B and C virus were negative, and a rapid test for human immunodeficiency virus (HIV) was also negative. Testing for autoantibodies such as for antinuclear antibody (ANA), antineutrophil cytoplasmic antibody (ANCA) was negative. Chest radiograph revealed no abnormality. His myeloma workup (urine BJP/serum M band), Lyme serology, and CSF veneral disease research laboratory were negative. A whole body FDG positron emission tomography-computed tomography (PET-CT) was also reported as normal. Magnetic resonance imaging (MRI) study of brain and spine with contrast was done, it revealed, short tau inversion recovery (STIR) hyperintensities of bilateral brachial plexus and lumbar plexus (-Fig. 1A, B), lumbosacral paraspinal muscles (myositis; - Fig. 2) and compression fracture of D12 vertebral body with minimal retropulsation ( - Fig. $\mathbf{3}$ ).

Since a detailed pyrexia of unknown origin (puo) evaluation was inconclusive, his history was revisited, and interestingly, he gave history of having worked in an abattoir in the recent past. In view of his occupational history, pyrexia of unknown origin of 6-month duration, chronic encephalitis with myeloradiculitis, vertebral and paravertebral muscle disease, a possibility of brucellosis was considered and serum brucella immunoglobulin (Ig) $\mathrm{G}$ and IgM antibodies (enzymelinked immunosorbent assay [ELISA]) were sent. His test revealed very high titers of both Brucella $\operatorname{IgG}(33.0 \mathrm{U} / \mathrm{mL})$ and Brucella IgM (76.2 U/ml) antibodies. A diagnosis of chronic neurobrucellosis was thus confirmed.

Our patient was treated with an oral combination of capsule doxycycline $100 \mathrm{mg}$ twice daily and capsule rifampicin $600 \mathrm{mg}$ once daily for 6 weeks, along with injection gentamicin $240 \mathrm{mg}$ once daily for the first 2 weeks. Supportive therapy included injection Human Mixtard subcutaneously for glycemic control and clean intermittent catheterization for spastic bladder symptoms. He was afebrile after 2 weeks of therapy with no recurrence of encephalopathy. He also had improvement in lower limb power. At 3 months of follow-up, he was ambulating safely with one stick support but the spastic bladder symptoms persisted, requiring regular clean intermittent cathetrization.

\section{Discussion}

In a tropical country like India, where pyrexia of unknown origin is a challenge for clinicians, three following features distinguish brucellosis from other tropical fevers: an undulating pattern of fever in untreated/partially treated patients, severe rheumatism in almost half of all patients, and malodorous perspiration.

Brucellosis can affect every organ; however, the commonest sites involved are osteoarticular/musculoskeletal. Second most common site of involvement is the reproductive system. Other systems involved are the hematological system, cardiopulmonary, and genitourinary systems. ${ }^{3.4} \mathrm{CNS}$ involvement is rare with brucella infection and when present, it is associated
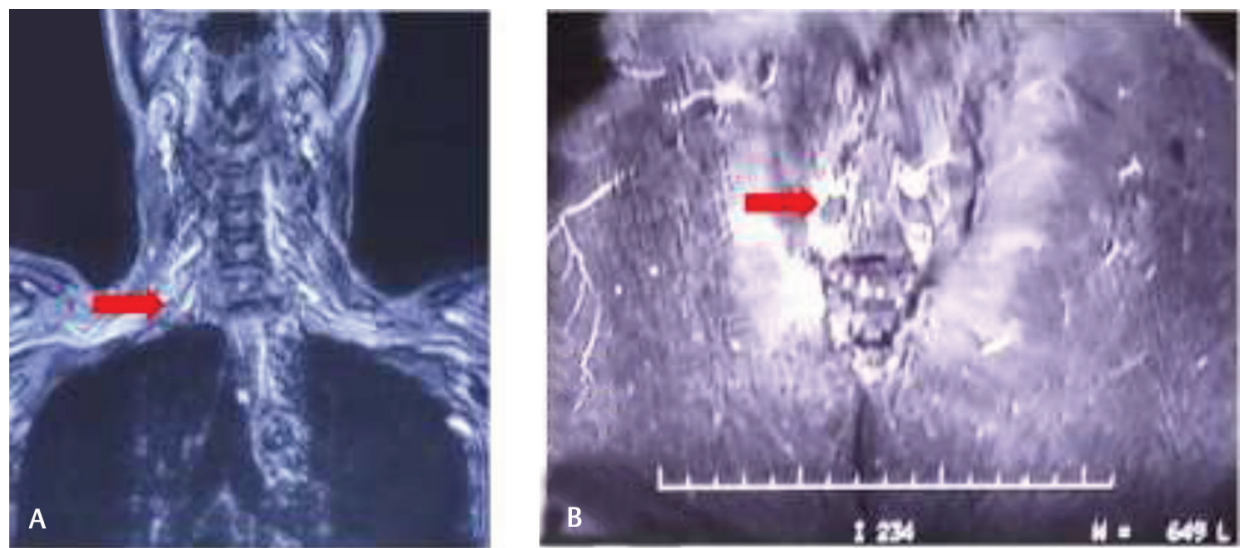

Fig. 1 (A and B) STIR edema (red arrow) involving nerves of brachial plexus and the lumbar plexus. STIR, short tau inversion recovery. 


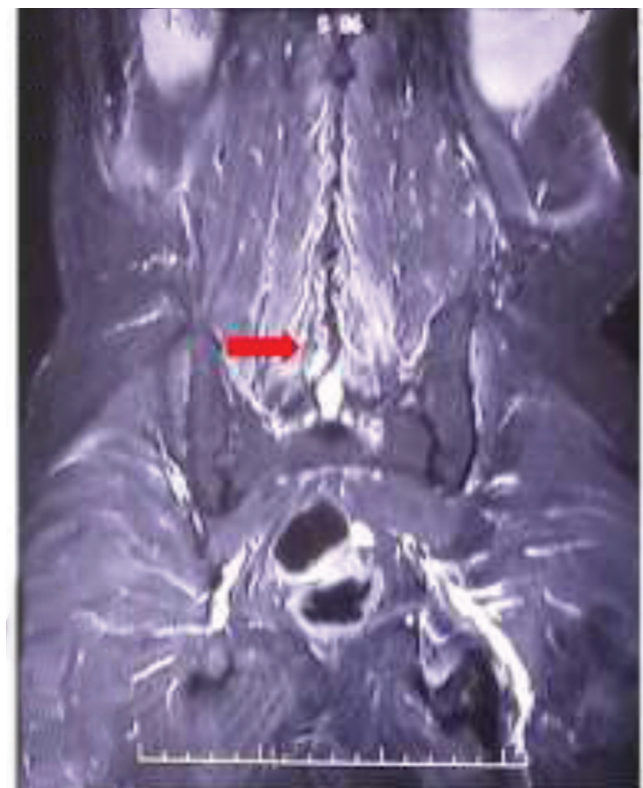

Fig. 2 STIR edema involving paraspinal muscles (red arrow). STIR, short tau inversion recovery.

with a bad prognosis. It usually manifest with meningitis, encephalitis, meningovascular disease, brain abscesses, or demyelinating syndromes. ${ }^{3,6}$ Myelitis or myeloradiculopathy is very rare to happen and reported in only 1.2 to $3 \%$ cases of neurobrucellosis. ${ }^{6}$ Brucella causes involvement of spinal cord by various mechanisms, which include infectious process itself, immune mechanisms, septic embolization, or venous thrombosis due to brucella vasculitis. Encephalopathy in brucellosis is thought to be due to vascular involvement. ${ }^{6}$ Our patient had rare manifestations of both encephalopathy and myeloradiculopathy.

As per Ceran et al, in a case series of 18 patients, only 3 (16.6\%) patients had myelitis and associated meningitis, but there was not a single case of myeloradiculopathy which was observed in our case. ${ }^{5}$ To our knowledge, there is only one Indian case report of neurobrucellosis from Himachal Pradesh where transverse myelitis was the presenting features. ${ }^{7}$

As per Tali et al, MRI spine may show normal cord signals or nonspecific changes during acute phases of Brucellosis myelopathy. ${ }^{6}$ This was the finding in our patient too, where clinically he had clear signs of a myelopathy, but his MRI spine did not show any cord compression or cord signal change.

History of travel through a brucellosis endemic region, working in a microbiology laboratory, consumption of unpasteurized milk or cheese, and close contact with domestic animals, all help in diagnosis of brucellosis. ${ }^{3,4}$ Our patient was an abattoir worker and belonged to Dharmpuri district of Tamil Nadu which is known to be endemic for brucellosis.

Since brucellosis has a very variable clinical presentation, the diagnosis needs a clear history of some form of exposure to animals or animal products along with a consistent disease pattern. In developing countries like India only serological tests are available. These tests detect antibodies by agglutination tests (tube or plate methods) and ELISA test. In endemic

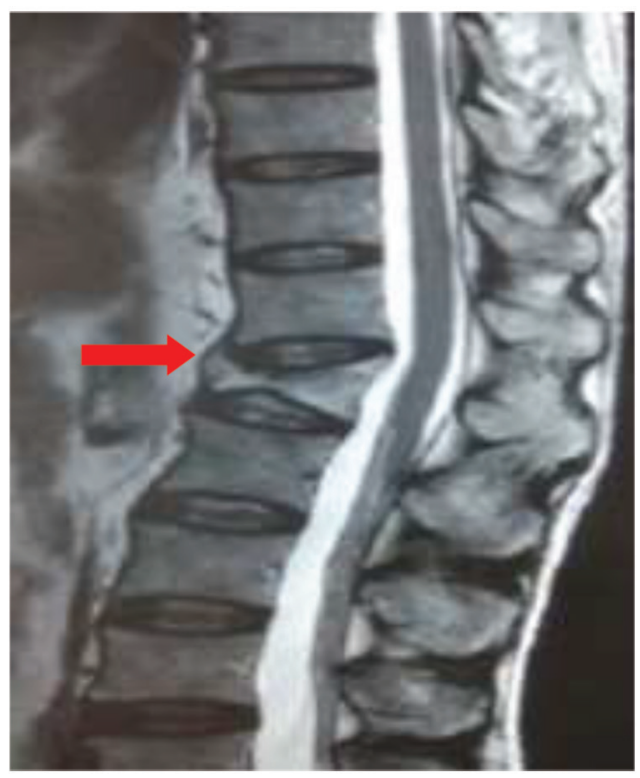

Fig. 3 T2WI MRI dorsal spine-sagittal view compression fracture of D12 vertebrae (red arrow). MRI, magnetic resonance imaging; T2WI, T2-weighted.

areas, agglutination test titers of 1:320 to 1:640 or higher are considered clinically significant; in nonendemic areas, a lower titer of $\geq 1: 160$ is also considered significant. Demonstrating rising titers on a repeat testing after 2 to 4 weeks, can also establish the diagnosis when a single test is inconclusive. ELISA for IgG and IgM antibody is 90\% Sensitive, 95\% specific and used for definitive diagnosis in the settings of history of exposure and consistent clinical findings. ${ }^{3,4}$ Since the immune response in brucella may be delayed in some cases, whenever there is a strong clinical suspicion, it is worthwhile repeating the antibody titers after 1 to 2 weeks.

A combination of synergistic antibiotics is needed for treatment of brucellosis.,4 Therapy of acute systemic brucellosis ( $<1$ month), requires a 6 -week course of combination antibiotic therapy. However complex or multifocal disease calls for a longer (8-12 weeks) course to achieve complete cure. A double drug regimen containing doxycycline and rifampin for 6 weeks is considered quite effective. Or else an injectable regimen with streptomycin ( $1 \mathrm{~g} /$ day intramuscular for 2 weeks) or gentamicin (240 mg intramuscular for 2 weeks) can also be used. ${ }^{8}$ A three-drug regimen containing doxycycline and rifampin for 6 weeks, combined with an initial 2-week course of a parenteral aminoglycoside (gentamicin/streptomycin) was found to be superior to double-drug regimens, in a recent meta-analysis. ${ }^{9}$

\section{Conclusion}

This case brings out the significance of occupational history taking in clinical medicine. Chronic encephalomyelitis with paraparesis is an unusual presentation of neurobrucellosis. In systemic brucellosis, sometimes bone and muscle diseases maybe clinically silent and evident only on imaging, as was the finding this case. Brucellosis associated myelitis is multifactorial, and MRI spine can be normal in such forms of infectious myelitis. When all exhaustive tests are normal in a PUO, 
even in today's era of modern diagnostics, one has to fall back on a good history to achieve a correct diagnosis!

\section{Conflict of Interest}

None declared.

\section{References}

1 Pappas G, Papadimitriou P, Akritidis N, Christou L, Tsianos EV. The new global map of human brucellosis. Lancet Infect Dis 2006;6(2):91-99

2 Mantur BG, Amarnath SK. Brucellosis in India - a review. J Biosci 2008;33(4):539-547

3 Pappas G, Akritidis N, Bosilkovski M, Tsianos E. Brucellosis. N Engl J Med 2005;352(22):2325-2336

4 Beeching NJ, Corbel MJ, Brucellosis. In: Dennis L. Kasper, Anthony S. Fauci, Stephen L. Hauser, Dan L. Longo, J. Larry
Jameson JL, eds. Harrison' s Principles of Internal Medicine. 19th ed. McGraw-Hill; 2015; 194e Part 08:001-006

5 Ceran N, Turkoglu R, Erdem I, et al. Neurobrucellosis: clinical, diagnostic, therapeutic features and outcome. Unusual clinical presentations in an endemic region. Braz J Infect Dis 2011;15(1):52-59

6 Tali ET, Koc AM, Oner AY. Spinal brucellosis. Neuroimaging Clin N Am 2015;25(2):233-245

7 Raina S, Sharma A, Sharma R, Bhardwaj A. Neurobrucellosis: a case report from himachal pradesh, india, and review of the literature. Case Rep Infect Dis 2016;2016:2019535

8 Solís García del Pozo J, Solera J. Systematic review and meta-analysis of randomized clinical trials in the treatment of human brucellosis. PLoS One 2012;7(2):e32090

9 Skalsky K, Yahav D, Bishara J, Pitlik S, Leibovici L, Paul M. Treatment of human brucellosis: systematic review and meta-analysis of randomised controlled trials. BMJ 2008;336(7646):701-704 